\title{
Relation between thyroid hormone status and concomitant medication in hyperlipidemic patients with statin induced adverse effects
}

\author{
Eszter Berta ${ }^{1,3}$, Mariann Harangi ${ }^{2}$, Noémi Zsíros ${ }^{2}$, Endre V. Nagy ${ }^{1}$, György Paragh², Miklós Bodor ${ }^{1,3}$
}

${ }^{1}$ Division of Endocrinology, ${ }^{2}$ Division of Metabolic Diseases, ${ }^{1}$ Department of Clinical Pharmacology, University of

Debrecen, Hungary

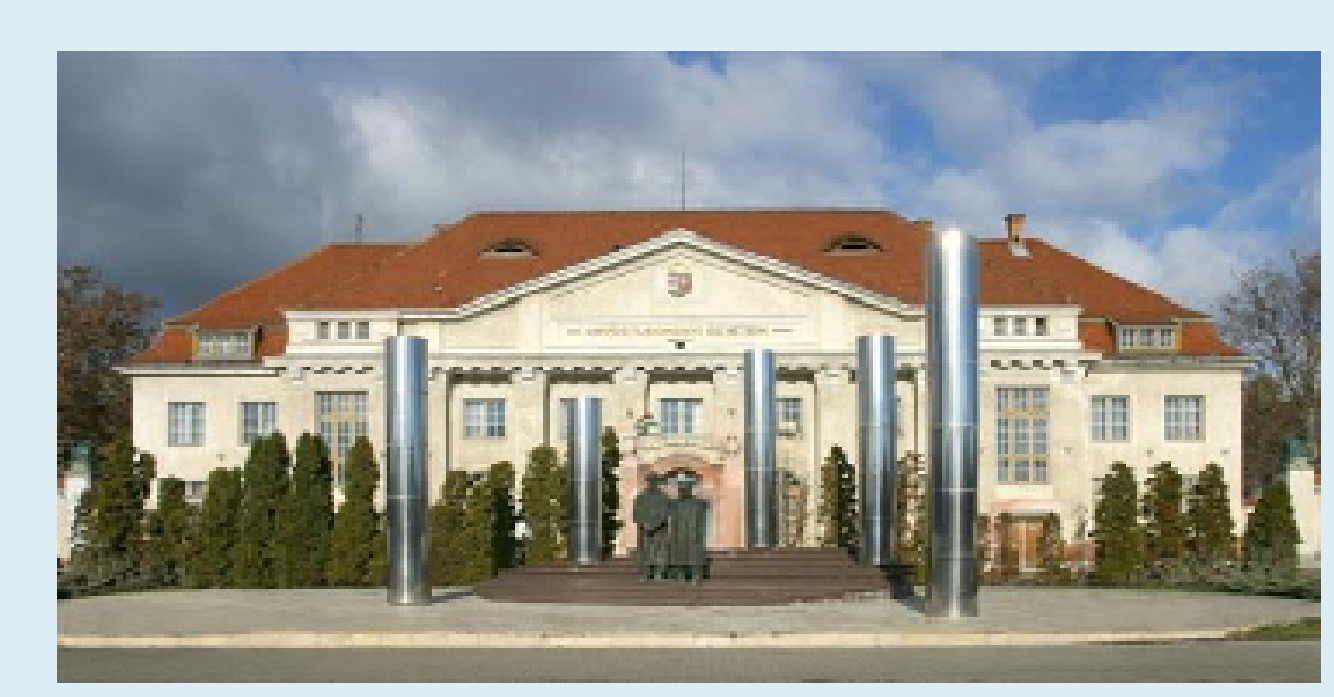

INTRODUCTION

Statins are effective treatment for the prevention of cardiovascular diseases and used extensively worldwide. However, adverse effects induced by statins including myopathy, hepatopathy and gastrointestinal conplains are the major barrier of maximalizing cardiovascular risk reduction.

Hypothyroidism and administration of drugs metabolized on the same cytochrome P450 (CYP) pathways where statin biotransformation occurs (CYP3A4, 2C9 and 2C19) represent a significant risk factor for statin induced adverse effects especially myopathy.

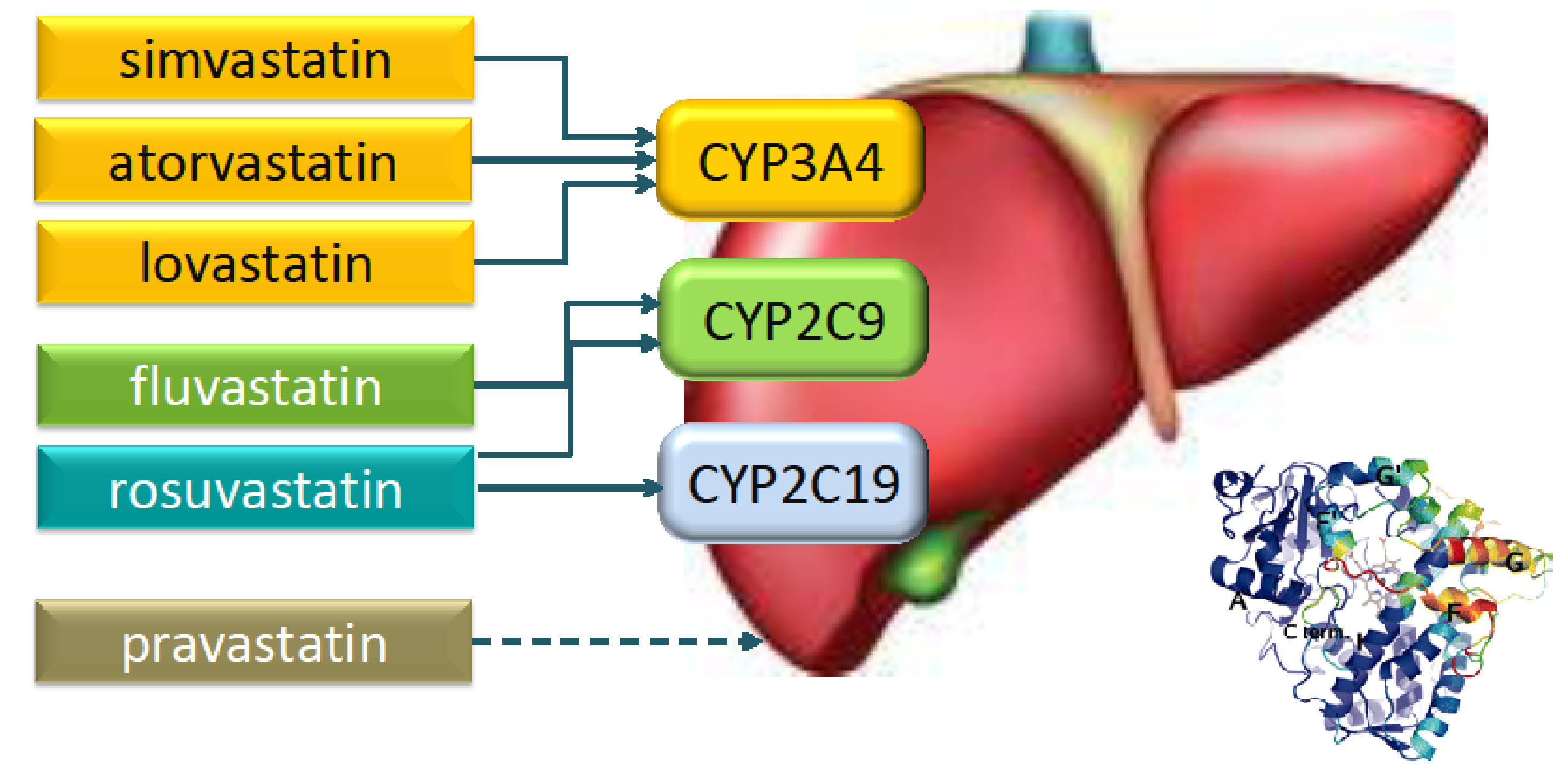

\section{OBJECTIVES}

We investigated the levels of the free thyroid hormones and CYP metabolism of concomitant medication in 101 hyperlipidemic patients (Table 1) with statin induced adverse effects including myopathy (56 cases; $55.4 \%$ ), hepatopathy (39 cases; $38.6 \%$ ) and gastrointestinal adverse effects ( 24 cases; $23.8 \%$ ).

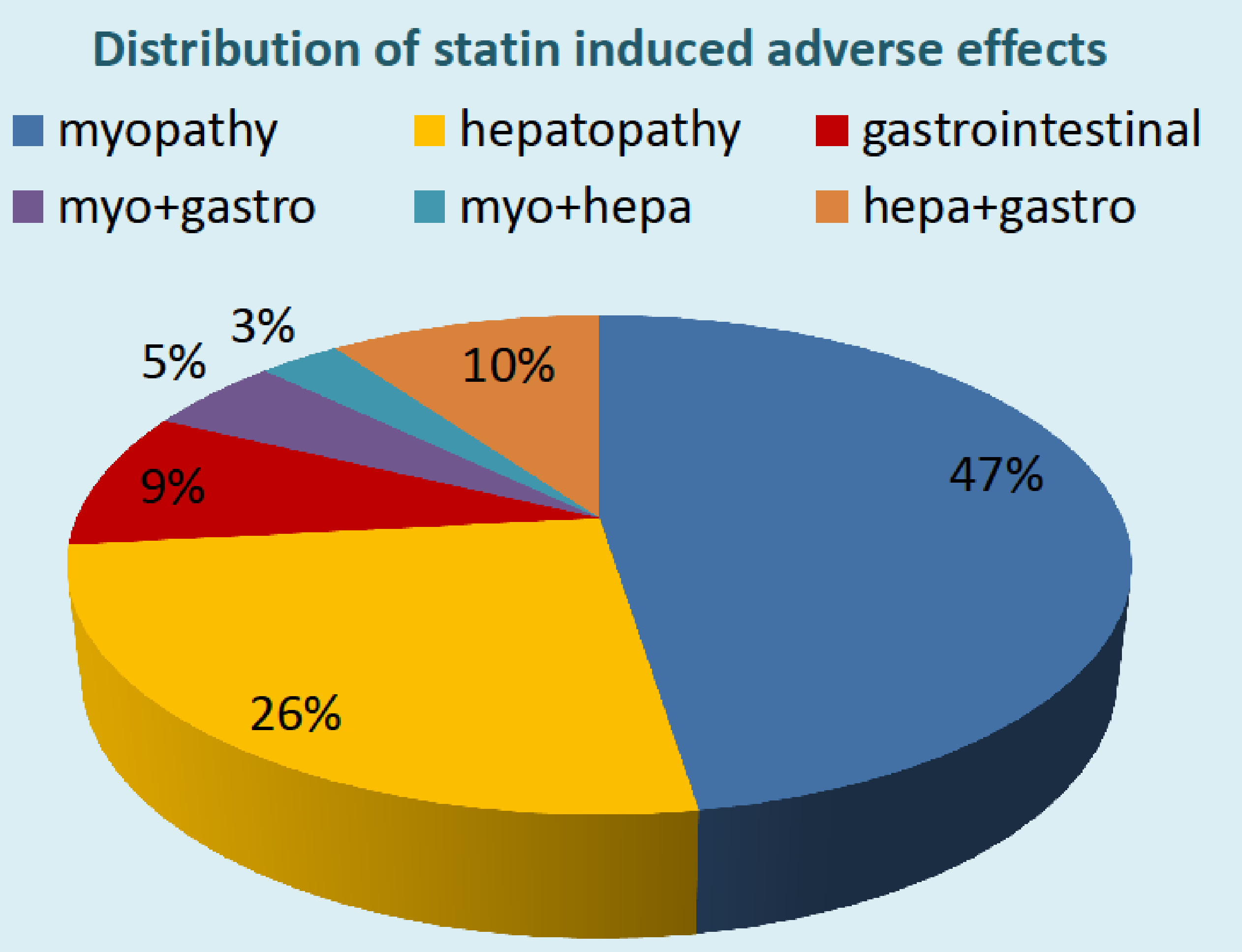

PATIENTS AND METHODS

Patients were recruited at the Department of Medicine, University of Debrecen. Informed consent was obtained from all patients after explaining the nature and the purpose of the study.

Table 1. Laboratory parameters and anthropometric data of the patients with and without statin induced myopathy

\begin{tabular}{llll}
\hline & Myopathy $(\mathrm{n}=56)$ & $\begin{array}{l}\text { No myopathy } \\
(\mathrm{n}=55)\end{array}$ & $\begin{array}{l}\text { All patients } \\
(\mathrm{n}=101)\end{array}$ \\
\hline Age (years) & $62.6 \pm 8.9$ & $59.5 \pm 10.9$ & $61.3 \pm 9.9$ \\
BMI (kg/m2) & $28.9 \pm 4.0$ & $27.2 \pm 4.5$ & $28.2 \pm 4.29$ \\
Waist circumference (cm) & $101.6 \pm 10.9$ & $94.0 \pm 11.6$ & $98.5 \pm 11.7$ \\
\hline Total cholesterol (mmol/l) & $7.33 \pm 1.57$ & $7.63 \pm 1.69$ & $7.46 \pm 1.62$ \\
LDL-C (mmol/l) & $4.64 \pm 1.59$ & $4.66 \pm 1.45$ & $4.65 \pm 1.52$ \\
HDL-C (mmol/l) & $1.39 \pm 0.4$ & $1.53 \pm 0.44$ & $1.45 \pm 0.43$ \\
Triglyceride (mmol/l) & $2.45(1.6-4.4)$ & $2.4(1.6-4.3)$ & $2.43(1.5-4.3)$ \\
ApoB (g/l) & $1.30 \pm 0.35$ & $1.31 \pm 0.36$ & $1.30 \pm 0.35$ \\
ApoA (g/l) & $1.60 \pm 0.32$ & $1.72 \pm 0.41$ & $1.65 \pm 0.36$ \\
CRP (mg/l) & $3.11(1.6-5.8)$ & $2.67(1.7-8.1)$ & $2.79(1.7-5.8)$ \\
Creatine kinase (U/l) & $198(121-308)$ & $94(67-128)^{*}$ & $131.5(87-235)$ \\
\hline
\end{tabular}

Table 2. Thyroid hormone levels of the patients with and without statin induced myopathy

\begin{tabular}{llll}
\hline & Myopathy $(n=56)$ & No myopathy $(n=55)$ & All patients $(n=101)$ \\
\hline STSH & $5.05 \pm 16.35$ & $2.09 \pm 2.26$ & $3.78 \pm 12.5$ \\
fT3 & $5.1 \pm 2.38$ & $4.74 \pm 0.96$ & $4.94 \pm 1.88$ \\
fT4 & $15.38 \pm 4.8$ & $14.55 \pm 4.3$ & $15.2 \pm 4.58$ \\
\hline
\end{tabular}

RESULTS AND DISCUSSION I.

During our one-year follow-up we found abnormal thyroid hormone levels in 5 patients presenting with statin-induced side effects (4.95\%); clinical hypothyroidism in 2 and hyperthyroidism in 3 cases. These data are in concordance with the incidence of thyroid dysfunction in the normal population. 11 patients had a positive history for hypothyroidism (10.9\%). This result is slightly higher than the one expected in the normal population (2.8-7.5\%). The difference might be explained by the fact that these patients were on levothyroxin substitution, which drug is metabolised also on CYP $3 \mathrm{~A} 4$ and as a result the risk of statin-induced sideeffects among hyperlipidemic patients might increase. Myopathy occurred in one patient with hypo- and in two patients with hyperthyroidism. There were no significant differences in the TSH, fT4 and fT3 levels between patients with statin induced myopathy or other types of adverse effects (Table 2).

Table 3. Number of patients using drugs and number of drugs metabolized by CYP isoforms also used by statins

\begin{tabular}{lllllll}
\hline & CYP3A4 & $\%$ & CYP2C9 & $\%$ & CYP2C19 & $\%$ \\
\hline patients & 66 & 65.3 & 67 & 66.3 & 54 & 53.5 \\
drugs & 147 & & 128 & & 81 & \\
\hline
\end{tabular}

Ratio of statin users in patients with and without myopathy

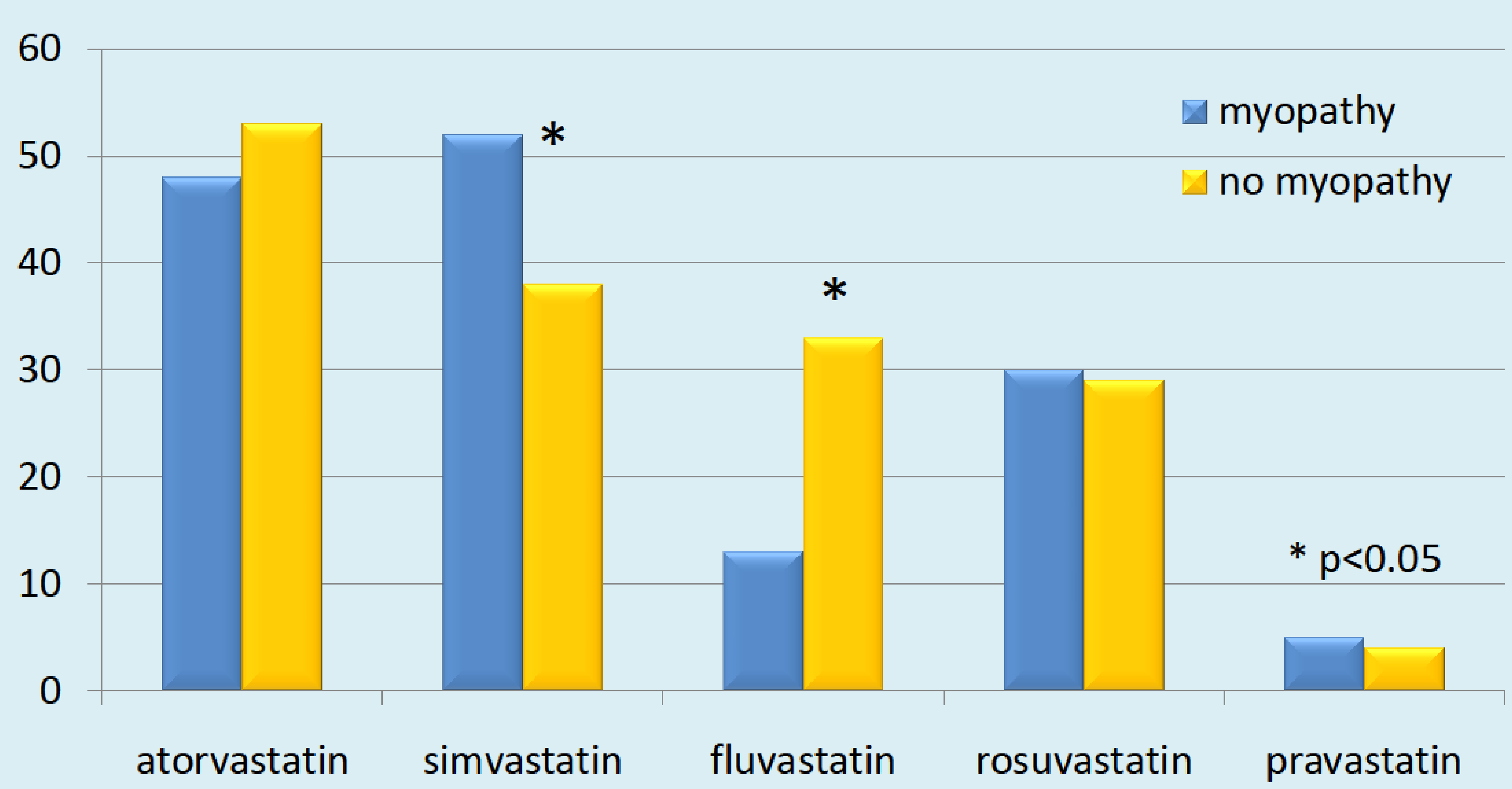

RESULTS AND DISCUSSION II.

In our study $77.2 \%$ of the patients with adverse effects caused by lipid-lowering therapy were administered drugs for the treatment of coexisting illnesses metabolized by CYP isoforms also used by statins (Table 3). Patients with myopathy took significantly more drugs metabolized by CYP3A4 compared to patients with other types of adverse effects $(p<0.05)$. More myopathy cases were found in patients on simvastatin treatment compared to other statin administrations, probably due to the competition between the HMG-CoA reductase inhibitors and other drugs on CYP3A4 (52\% vs. $38 \%$, ns.). In contrast, significantly less patients with myopathy were on fluvastatin treatment $(13 \%$ vs. $33 \%, p<0.05)$ compared to patients with other types of statin induced adverse effects, most likely because of the different metabolization pathway of this antihyperlipidemic drug.

\section{CONCLUSIONS}

Both abnormal thyroid hormone status and concomitant administration of drugs metabolized by CYP3A4, 2C9 and 2C19 enzymes that are responsible for the first step degradation of the widely used lipid-lowering agents are relatively frequent among our patients with statin induced adverse effects. Normalizing the thyroid hormone status and selecting the most suitable concomitant medication with respect to the known metabolization pathways may reduce the risk of statin induced adverse effects. 